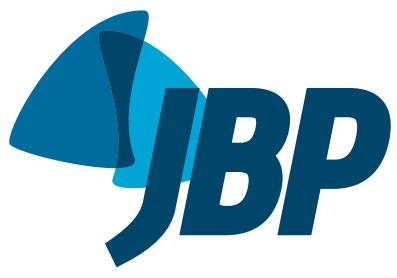

\title{
Lung ultrasound assessment of response to antibiotic therapy in cystic fibrosis exacerbations: a study of two cases
}

\author{
Andressa Oliveira Peixoto1,2,3,a, Fernando Augusto Lima Marson, 1,2,4,5, \\ Tiago Henrique Souza ${ }^{1,6, c}$, Andrea de Melo Alexandre Fraga1,3,d, \\ José Dirceu Ribeiro ${ }^{1,3, e}$
}

\section{TO THE EDITOR}

Cystic fibrosis (CF) pulmonary exacerbations (PEx) cause approximately $50 \%$ of the decline in lung function. ${ }^{(1)}$ Although there is no consensus, the criteria for defining PEx currently consist of abnormal sputum and/or chest X-ray findings; anorexia; increased cough; dyspnea; fatigue/lethargy; fever; hemoptysis; decreased overall health status; > 10\% decrease in $\mathrm{FEV}_{1}$; and weight loss.(2) However, two questions remain unanswered ${ }^{(1)}$ : how can we improve the treatment of PEx and which imaging techniques can indicate PEx and/or assess pulmonary involvement? Within this context, we can highlight the use of lung ultrasound (LUS), which is a rapid, radiation-free method that is easily reproducible, widely available, and low cost. LUS can be useful for assessing PEx and response to antibiotic therapy. Experimentally, we used LUS before and after antibiotic therapy in two female CF patients who had PEx. The female CF patients had two sweat chloride results $\geq 60 \mathrm{mEq} / \mathrm{L}$ and two pathogenic variants in the CFTR gene. The following assessments were performed: completion of a clinical/ demographic questionnaire; spirometry; chest HRCT on the day of the first LUS was performed; use of the Bhalla CT scoring system; measurement of $\mathrm{SpO}_{2}$; LUS; and routine sputum culture (Table 1 ). This study was approved by the local research ethics committee (CAAE no. 64515817.4.0000.54.04).

According to the international recommendation for point-of-care LUS, this test can detect the presence of A-pattern (normal lung sliding and regular pleural line echogenicity with a predominance of A-line artifacts) or B-pattern (presence of at least three B-line artifacts) per lung region. ${ }^{(3,4)}$ In our study, the lung was divided into 12 regions. The physical and anatomical nature of B-lines ("comet tail" artifacts; hyperechoic, vertical lines that mask A-lines originating from the visceral pleura and move with lung sliding) is not fully understood; however, their occurrence is associated with the presence of hydrostatic and/or inflammatory fluid in the lung interstitium. ${ }^{(4)}$ In addition, LUS can identify consolidation (hypoechoic subpleural area with irregular margins and heterogeneous texture, possibly with a hyperechoic image inside and/or B-lines adjacent to its posterior margin or an aspect similar to that of the liver parenchyma) and pleural effusion (anechoic space between the visceral and parietal pleura). ${ }^{(3)}$ Patient 1 (CFTR genotype, F508del/ G542X) met the following criteria for PEx: increased cough; increased sputum production and change in sputum appearance/consistency; worsening of findings on pulmonary auscultation; and positive routine culture for Staphylococcus aureus and Achromobacter xylosoxidans. During follow-up, oral antibiotic therapy was prescribed for 15 days. The Shwachman-Kulczycki (SK) score ${ }^{(5)}$ indicated lack of resistance and end-of-day tiredness, but good school attendance (general activity domain); presence of obstructive pulmonary disease, infection, lobular atelectasis, and bronchiectasis (radiological findings domain); weight and height around the 25th percentile, good muscle mass and tone, and well-formed, near-normal stools (nutrition domain); and no cough, normal heart and respiratory rates, clear lungs, and good posture (physical examination domain). In the assessment of PEx, we considered the patient's or caregiver's report of increased cough, increased sputum production, and/ or change in sputum appearance/consistency. However, in the SK score, ${ }^{(5)}$ the presence of cough was assessed during the visit, and this information was different from what was reported previously. In summary, the total SK score was 75, classified as "good". LUS assessment showed that, in four lung regions, the pattern changed from $B$ to $A$ after antibiotic therapy, being classified as a mixed pattern at both time points (Table 1 ).

Patient 2 (CFTR genotype, F508del/F508del) met the following criteria for PEx: increased cough; increased sputum production and change in sputum consistency; tiredness; intolerance to physical exertion; weigh loss; decreased $\mathrm{SpO}_{2}$; and positive routine culture for mucoid and nonmucoid Pseudomonas aeruginosa. During

1. Departamento de Pediatria, Faculdade de Ciências Médicas, Universidade Estadual de Campinas, Campinas (SP) Brasil.

2. Centro de Investigação em Pediatria, Faculdade de Ciências Médicas, Universidade Estadual de Campinas, Campinas (SP) Brasil.

3. Unidade de Urgência e Emergência, Hospital de Clínicas, Faculdade de Ciências Médicas, Universidade Estadual de Campinas, Campinas (SP) Brasil.

4. Departamento de Genética Médica e Medicina Genômica, Faculdade de Ciências Médicas, Universidade Estadual de Campinas, Campinas (SP) Brasil.

5. Programa de Pós-Graduação em Ciências da Saúde, Universidade São Francisco, Bragança Paulista (SP) Brasil.

6. Unidade de Terapia Intensiva Pediátrica, Hospital de Clínicas, Faculdade de Ciências Médicas, Universidade Estadual de Campinas, Campinas (SP) Brasil. Financial support: The following authors received financial support: Peixoto AO, Conselho Nacional de Desenvolvimento Cientifico e Tecnológico (CNPq, National Council for Scientific and Technological Development; Grant no. 407364/2016-1); Marson FAL, Fundação de Amparo à Pesquisa do Estado de São Paulo (FAPESP, São Paulo Research Foundation; Grant nos. 2011/12939-4, 2011/18845-1, 2015/12183-8, and 2015/12858-5) and State University at Campinas Fundo de Apoio à Pesquisa ao Ensino e à Extensão (FAEPEX, Fund for the Support of Research, Teaching, and Extension; Grant no. 0648/2015); and Ribeiro JD FAPESP (Grant nos. 2011/18845-1 and 2015/12183-8) and CNPq (Grant no. 407364/2016-1)

a. (iD) http://orcid.org/0000-0002-8407-4087; b. (iD) http://orcid.org/0000-0003-4955-4234; c. (iD) http://orcid.org/0000-0001-6944-0221;

d. (iD http://orcid.org/0000-0003-0999-5350; e. (iD http://orcid.org/0000-0002-3387-5642 
follow-up, oral antibiotic therapy was prescribed for 15 days. The SK score ${ }^{(5)}$ indicated lack of resistance and end-of-day tiredness, but good school attendance (general activity domain); presence of obstructive pulmonary disease, infection, lobular atelectasis, and bronchiectasis (radiological findings domain); weight and height below the 3rd percentile, weak muscle tone, reduced muscle mass, mild/moderate abdominal distention, and voluminous, greasy, poorly-formed stools (nutrition domain); frequent cough, usually productive, chest retraction, moderate emphysema, chest deformity, frequent crackles, and digital clubbing (physical examination domain). The total SK score was 45 , classified as "moderate". LUS assessment showed that, in one lung region, the pattern changed from $B$ to A after antibiotic therapy, being classified as a mixed pattern at both time points (Table 1 ).

Both patients were chronically colonized/infected with the aforementioned bacteria and had changes in routine culture results from before to after antibiotic therapy.

The LUS images were examined by a pulmonologist with specific training in LUS interpretation. A second member of the team who specialized in radiology analyzed the LUS findings in a blind fashion. Both professionals scored the LUS images and interpreted the image findings, with identical results. The ultrasound scoring system and the full description of the methods have been published elsewhere. ${ }^{(6)}$

In reviewing the literature, there is an evident need for a test for assessing antibiotic therapy success in PEx. In addition, there is no consensus regarding the criteria defining the start/end of PEx and the time required for treatment. Furthermore, it is not always possible to identify who will require short-course antibiotic therapy (10-14 days, early responders) or long-course antibiotic therapy (approximately 21 days, late responders). ${ }^{(1,7)}$ Although clinical markers and pulmonary function test results have been used as tools to assess response to treatment, they have limitations and lose specificity as the disease progresses. ${ }^{(1)}$ In this context, our report encourages the applicability of LUS for assessing PEx.

In CF, PEx are markers of disease progression and should be monitored during routine visits. We know that CF patients have experienced an increase in survival despite chronic airway colonization with

Table 1. Data from cystic fibrosis patients and from lung ultrasound assessments before and after antibiotic therapy.

\begin{tabular}{|c|c|c|c|c|}
\hline \multirow[t]{2}{*}{ Data } & \multicolumn{2}{|c|}{ Patient 1} & \multicolumn{2}{|c|}{ Patient 2} \\
\hline & Before & After & Before & After \\
\hline Age, years & \multicolumn{2}{|c|}{22} & \multicolumn{2}{|c|}{18} \\
\hline $\mathrm{BMI}, \mathrm{kg} / \mathrm{m}^{2}$ & \multicolumn{2}{|c|}{23.34} & \multicolumn{2}{|c|}{17.41} \\
\hline $\mathrm{SpO}_{2}$ & \multicolumn{2}{|c|}{95} & \multicolumn{2}{|c|}{92} \\
\hline Comorbidities & \multicolumn{2}{|c|}{ PIns } & \multicolumn{2}{|c|}{ PIns, DM } \\
\hline CFTR & \multicolumn{2}{|c|}{ F508del/G542X } & \multicolumn{2}{|c|}{ F508del/F508del } \\
\hline FVC, \% predicted & 73 & 74 & 38 & 39 \\
\hline $\mathrm{FEV}_{1}, \%$ predicted & 55 & 57 & 42 & 41 \\
\hline $\mathrm{FEV}_{1} / \mathrm{FVC}$ & 75 & 77 & 99 & 94 \\
\hline $\mathrm{FEF}_{25-75 \%}, \%$ & 24 & 26 & 53 & 46 \\
\hline Bhalla $^{a}$ & \multicolumn{2}{|c|}{21} & \multicolumn{2}{|c|}{24} \\
\hline \multicolumn{5}{|c|}{ Regions assessed on LUS } \\
\hline 1 & A & A & B & $A+P I$ \\
\hline 2 & $\mathrm{C}+\mathrm{PI}$ & $\mathrm{C}+\mathrm{PI}$ & $B+C$ & $B+C$ \\
\hline 3 & B & A & B & B \\
\hline 4 & $\mathrm{~B}+\mathrm{PI}$ & $\mathrm{B}+\mathrm{PI}$ & B & B \\
\hline 5 & $A+P I$ & $A$ & B & B \\
\hline 6 & B & $A$ & $B+C$ & $B+C$ \\
\hline 7 & $A$ & A & B & B \\
\hline 8 & B & B & B & B \\
\hline 9 & $\mathrm{~A}+\mathrm{PI}$ & A & $B+C$ & $B+C$ \\
\hline 10 & B & A & $\mathrm{B}+\mathrm{PI}$ & $\mathrm{B}+\mathrm{PI}$ \\
\hline 11 & A & A & B & B \\
\hline 12 & B & A & B & B \\
\hline Score ${ }^{b}$ & $8 / 36$ & $4 / 36$ & $18 / 36$ & $17 / 36$ \\
\hline
\end{tabular}

BMI: body mass index; PIns: pancreatic insufficiency; DM: diabetes mellitus; CFTR: cystic fibrosis transmembrane regulator; LUS: lung ultrasound; A: A-pattern; B: B-pattern; PI: pleural irregularity; and C: consolidation. ${ }^{\text {aThe }}$ modified Bhalla CT scoring system was used as in Folescu et al.(10): the total score for each patient is obtained by summing the scores for each morphological change, which are attributed on the basis of the severity/extent of the abnormality. The total score can range from zero (absence of abnormalities) to 37 (all abnormalities present and severe). 'The higher the proportion value, the greater the pulmonary involvement in the area, with the presence of consolidation being assigned 2 points and the presence of B-pattern being assigned 1 point. Therefore, the maximum score is 36 points. 
bacteria exhibiting increased drug resistance, which culminates in the use of numerous drugs (antibiotics and anti-inflammatory drugs) that require frequent monitoring, since we move toward personalized and precision medicine. ${ }^{(8,9)}$ In this process, LUS could be a tool that accompanies patients in determining their individual response to therapy, without causing complications or exposing patients to radiation.

In summary, LUS could be a useful tool to assess changes due to PEx and response to antibiotic therapy in CF. However, further studies involving a larger sample size are needed in order to confirm our findings, since only one of the two CF patients assessed had distinctly different LUS results before and after antibiotic therapy.

\section{AUTHOR CONTRIBUTION}

AOP conceived the project, collected the participant data, and drafted and revised the manuscript; FALM and JDR conceived the study and drafted and revised the manuscript; THS validated the results; and AMAF clinically assessed the study participants and validated the phenotypic findings against reproducibility criteria. All authors read and approved the final version of the article for submission.

\section{REFERENCES}

1. Schechter MS. Reevaluating approaches to cystic fibrosis pulmonary exacerbations. Pediatr Pulmonol. 2018;53(S3):S51-S63. https://doi. org/10.1002/ppul.24125

2. Fuchs HJ, Borowitz DS, Christiansen DH, Morris EM, Nash ML, Ramsey BW, et al. Effect of aerosolized recombinant human DNase on exacerbations of respiratory symptoms and on pulmonary function in patients with cystic fibrosis. The Pulmozyme Study Group. N Engl J Med. 1994;331(10):637-42. https://doi.org/10.1056/ NEJM199409083311003

3. Volpicelli G, Caramello V, Cardinale L, Mussa A, Bar F, Frascisco MF. Detection of sonographic B-lines in patients with normal lung or radiographic alveolar consolidation. Med Sci Monit. 2008;14(3):CR122-8.

4. Shwachman $H$, Kulczycki LL. Long term study of one hundred five patients with cystic fibrosis. Am J Dis Child. 1958;96:6-15.

5. Volpicelli G, Elbarbary M, Blaivas M, Lichtenstein DA, Mathis G, Kirkpatrick AW, et al. International evidence-based recommendations for point-of-care lung ultrasound. Intensive Care Med. 2012;38(4):57791. https://doi.org/10.1007/s00134-012-2513-4

6. Peixoto $\mathrm{AO}$. The use of ultrasound as a tool to evaluate pulmonary disease in cystic fibrosis [dissertation]. Campinas: Universidade Estadual de Campinas; 2019. Available from: http://repositorio. unicamp.br/jspui/bitstream/REPOSIP/334182/1/Peixoto_ AndressaOliveira_M.pdf

7. Flume PA, Mogayzel PJ Jr, Robinson KA, Goss $\mathrm{CH}$, Rosenblatt RL, Kuhn RJ, et al. Cystic fibrosis pulmonary guidelines: treatment of pulmonary exacerbations. Am J Respir Crit Care Med. 2009;180(9):802-8. https://doi.org/10.1164/rccm.200812-1845PP

8. Marson FAL, Bertuzzo CS, Ribeiro JD. Personalized or Precision Medicine? The Example of Cystic Fibrosis. Front Pharmacol. 2017:8:390. https://doi.org/10.3389/fphar.2017.00390

9. de Lima Marson FA, Bertuzzo CS, Ribeiro JD. Personalized Drug Therapy in Cystic Fibrosis: From Fiction to Reality. Curr Drug Targets. 2015;16(9):1007-17. https://doi.org/10.2174/138945011566614112 8121118

10. Folescu TW, Marques Ede A, Boechat MC, Daltro P, Higa LY, Cohen RW. High-resolution computed tomography scores in cystic fibrosis patients colonized with Pseudomonas aeruginosa or Staphylococcus aureus. J Bras Pneumol. 2012;38(1):41-9. https://doi.org/10.1590/ S1806-37132012000100007 